\title{
Composto de resíduos da fabricação de papel e celulose na produção de mudas de eucalipto
}

\author{
Fábio H. S. F. de Toledo ${ }^{1}$, Nelson Venturin², Leandro Carlos ${ }^{3}$, Bruna A. S. Dias ${ }^{4}$, \\ Regis P. Venturin ${ }^{5} \&$ Renato L. G. Macedo ${ }^{2}$
}

${ }^{1}$ Programa de Pós-Graduação em Recursos Florestais/Universidade de São Paulo. Piracicaba, SP. E-mail: fhtoledo@usp.br (Autor correspondente)

${ }^{2}$ Departamento de Ciências Florestais/Universidade Federal de Lavras. Lavras, MG. E-mail: venturin@dcf.ufla.br; rlgrisi@dcf.ufla.br

${ }^{3}$ Instituto Federal Goiano. Rio Verde, GO. E-mail: 1cmaestro@gmail.com

${ }^{4}$ Departamento de Ciências Florestais/Universidade Federal do Piauí. Bom Jesus, PI. E-mail: brunapropri@yahoo.com.br

${ }^{5}$ Empresa de Pesquisa Agropecuária de Minas Gerais. Lavras, MG. E-mail: regis@epamig.br

\section{Palavras-chave:} composto orgânico viveiro florestal qualidade de mudas

\begin{abstract}
R E S U M O
Este trabalho foi realizado com o objetivo de testar substratos obtidos a partir da combinação de um composto orgânico oriundo de resíduos do processo de fabricação de celulose, com um substrato base de controle. O estudo foi realizado no viveiro florestal da Universidade Federal de Lavras utilizando-se sementes do híbrido Eucalyptus “urograndis". Foram testados seis tratamentos com doses crescentes de composto orgânico. O experimento foi implantado em delineamento inteiramente casualizado, com seis tratamentos, cinco repetições e 30 mudas por parcela. A avaliação dos substratos foi mensurada através da análise do crescimento das plantas, ao longo do tempo e de sua qualidade, ao final do processo de produção. Quanto às análises de crescimento e à qualidade das mudas, os tratamentos que continham 60 e $80 \%$ de composto orgânico apresentaram as melhores respostas. Concluiu-se que a produção de mudas de Eucalyptus "urograndis" com os substratos testados mostrou-se tecnicamente viável de acordo com as características e os índices analisados.
\end{abstract}

\section{Key words:}

organic compost

forest nursery

seedling quality

\section{Compost of residues of pulp and paper in the production of eucalyptus seedlings}

\begin{abstract}
A B S T R A C T
This work was performed aiming to test substrates obtained from the combination of an organic compound, derived from waste of manufacturing process of cellulose, with a base substrate for control. The study was conducted at the Forest Nursery of the Federal University of Lavras using seeds of the hybrid Eucalyptus "urograndis". Six treatments with increasing doses of organic compound were tested. The experiment was implemented in a completely randomized design, with six treatments, five replications and 30 seedlings per plot. The substrate evaluation was measured through the analysis of plant growth throughout the experiment and the seedlings qualities at the end of production process. As for the analysis of growth and quality of seedlings, the treatments with 60 and $80 \%$ of organic compound presented the bests response. It was concluded that the seedling production of Eucalyptus "urograndis" with the substrates analysed proved technically feasiable according to the analysed characteristics and indexes.
\end{abstract}




\section{INTRODUÇÃo}

O setor de papel e celulose brasileiro é destaque na área florestal no âmbito mundial como o quarto maior produtor de celulose, o nono maior produtor de papel, além de ser o $13^{\circ}$ maior mercado de consumidores per capita de papel, contando com um total de 220 empresas de celulose e papel com atividades em 540 municípios, localizados em 18 estados (BRACELPA, 2013). Estima-se que o setor florestal manteve, em 2013, cerca de 4,4 milhões de empregos divididos em empregos diretos (0,6 milhões), indiretos (1,3 milhões) e resultantes do efeito-renda (2,4 milhões). No mesmo ano a arrecadação de tributos foi de R \$ 7,6 bilhões e o valor bruto da produção florestal foi de R\$ 56,3 bilhões (ABRAF, 2013).

Nos processos de obtenção de seus produtos as indústrias do setor florestal normalmente geram resíduos os quais podem ter diferentes tipos de destino, sendo sua reutilização no ciclo de produção uma das disposições mais econômicas e ecologicamente corretas (Galbiatti et al., 2007; Santos et al., 2013).

A temática sobre utilização de resíduos na formação de substratos destinados à produção de mudas tem sido frequente na literatura nacional e internacional (Ribeiro et al., 2007). Tendo em vista a existência de diversos tipos de resíduos este assunto se torna ainda mais complexo devido às diferentes possibilidades de combinações entre os mesmos em que alguns exemplos, são: biossólidos (Assenheimer, 2009), bagaço de cana e torta de filtro (Freitas et al., 2010), couro de wet-blue (Daudt et al., 2007), resíduos da agroindústria de chá preto (Lima et al., 2007), entre outros. O assunto recebe grande destaque por dar melhor destino aos resíduos gerados no setor florestal, em especial as empresas do ramo de celulose e papel.

Nas empresas do setor de celulose e papel são gerados diversos tipos de resíduos tais como, os denominados "Dregs" e "Grits" oriundos do processo "Kraft" para a extração de celulose (Barretto, 2008); a lama de cal e o lodo orgânico resultante de tratamentos de efluentes líquidos (Barretto, 2008); as cascas de eucalipto provenientes do processo de descascamento (Mendonça et al., 2008); as cinzas advindas da queima de biomassa nas caldeiras para obtenção de energia (Gonçalves \& Stape, 2002), entre outros.

Estudos vêm sendo executados na linha de reutilização de resíduos para a produção de mudas de Eucalyptus sp. (Trigueiro \& Guerrini, 2003; Maeda et al., 2007; Paiva et al., 2009), nos quais a maioria foi realizada utilizando-se apenas um resíduo ou os testando de forma individua porém se notou, após pesquisa sobre a reutilização de resíduos no meio florestal, escassez de estudos que contemplam misturas desses resíduos na composição de substratos para produção de mudas de eucalipto, assim como estudo realizado por Barretto (2008). Desta forma, realizou-se este trabalho com o objetivo de testar substratos obtidos a partir da combinação de um composto orgânico, oriundo de resíduos do processo de fabricação de celulose, com o substrato rotineiramente utilizado na Universidade Federal de Lavras.

\section{Material e Métodos}

O experimento foi instalado no viveiro da Universidade Federal de Lavras localizado a $21^{\circ} 13^{\prime} 40^{\prime \prime} \mathrm{S}$ e $44^{\circ} 57^{\prime} 50^{\prime \prime} \mathrm{W}$ e altitude de 925 m. O clima da região é do tipo Cwb segundo classificação de Köppen; as médias anuais de precipitação e temperatura são, respectivamente, $1.529,5 \mathrm{~mm}$ e $19,4^{\circ} \mathrm{C}$. Foram utilizados tubetes de polipropileno de $55 \mathrm{~cm}^{3}$ e bandejas de 96 células. A densidade durante todo o experimento foi de 206 mudas $\mathrm{m}^{-2}$.

O substrato base usado como controle e para a combinação com o composto foi formado a partir da mistura de $40 \%$ de fibra de coco, $30 \%$ de esterco curtido, $20 \%$ de casca de arroz carboizado, $10 \%$ de vermiculita média e $4 \mathrm{~kg} \mathrm{~m}^{-3}$ de adubo de liberação lenta (19-6-10, 3-4 meses). Já o composto orgânico utilizado foi formado por cinco resíduos da fábrica de celulose, sendo eles: $58 \%$ de lodo, $9 \%$ de Dregs, $25 \%$ de casca de eucalipto, $3,4 \%$ de grits e $4,5 \%$ de cinzas. O processo para formação do composto foi realizado conforme os padrões da empresa fornecedora. Os tratamentos do experimento foram combinações do composto orgânico com o substrato base (Tabela 1).

O experimento foi organizado em delineamento inteiramente ao acaso, com seis tratamentos e cinco repetições, sendo o valor de cada repetição fruto da média da mensuração de 30 mudas. As mesmas foram dispostas alternadamente nas células das bandejas desde a semeadura até o final do experimento, aos 120 dias após a semeadura.

Após o enchimento dos tubetes foi realizada a semeadura utilizando-se aproximadamente oito sementes por tubete. Para a proteção das sementes foi utilizada uma fina cobertura de palha de arroz carbonizada. Os tubetes ficaram 30 dias em casa de sombra (sombrite 70\%) com irrigação automática $(0,44 \mathrm{~L}$ $\mathrm{m}^{-2}$ a cada hora), sendo que em três semanas após a germinação foi realizado o raleio das mudas permanecendo apenas o indivíduo mais vigoroso por tubete. Posteriormente, as mudas seguiram para o viveiro a pleno sol, também em sistema de irrigação automático $\left(4,3 \mathrm{~L} \mathrm{~m}^{-2}\right.$ três vezes ao dia), quando teve início a adubação de cobertura de macro e micronutrientes recomendados por Gonçalves \& Benedetti (2000). A análise química e a física dos tratamentos se encontram na Tabela 2.

As características mensuradas para aferir o crescimento das mudas foram a altura da parte aérea $(\mathrm{H})$ e o diâmetro do colo (D) a $0,5 \mathrm{~cm}$ de altura do substrato. Foi realizada a medição da $\mathrm{H}$ aos 30, 60, 90 e 120 dias após a semeadura utilizando-se uma régua milimétrica. A medição do D foi realizada aos 60 , 90 e 120 dias após a semeadura através de um paquímetro digital. Para aferir a qualidade das mudas foram calculados, ao final dos 120 dias de experimento, os índices IQD (índice de qualidade de Dickson), H/D (relação entre a altura e o diâmetro do colo), H/PSA (relação entre a altura e o peso da

Tabela 1. Porcentagem de substrato base e composto orgânico na composição dos tratamentos utilizados para o crescimento de Eucalyptus "urograndis" em viveiro

\begin{tabular}{ccc}
\hline Tratamento & Substrato base & \multicolumn{1}{c}{ Composto orgânico } \\
\cline { 2 - 3 } T0 & 100 & $(\%)$ \\
T1 & 80 & 20 \\
T2 & 60 & 40 \\
T3 & 40 & 60 \\
T4 & 20 & 80 \\
T5 & 0 & 100 \\
\hline
\end{tabular}


Tabela 2. Análise química dos tratamentos do experimento, realizada pelo Laboratório de Análises Agrícolas e Ambientais Ltda., segundo as Normas da Federação dos Institutos de Pesquisas e Análises Agrícolas da Alemanha

\begin{tabular}{cccccccc}
\hline Tratamentos & & T0 & T1 & T2 & T3 & T4 & T5 \\
$\mathrm{pH}$ & $\mathrm{H}_{2} \mathrm{O}$ & 6,90 & 7,40 & 7,70 & 7,70 & 7,90 & 8,00 \\
$\mathrm{~N}$ total & $\mathrm{mg} \mathrm{kg}^{-1}$ & 4099 & 5539 & 6494 & 4759 & 5285 & 4865 \\
$\mathrm{P}$ & $\mathrm{mg} \mathrm{kg}^{-1}$ & 482,8 & 245,5 & 45,8 & 25,4 & 15,5 & 17,2 \\
$\mathrm{~K}$ & $\mathrm{mg} \mathrm{kg}^{-1}$ & 3100 & 1590 & 1176 & 1011 & 1003 & 870 \\
$\mathrm{Ca}$ & $\mathrm{mg} \mathrm{kg}^{-1}$ & 168 & 52 & 64 & 56 & 81 & 96 \\
$\mathrm{Mg}$ & $\mathrm{mg} \mathrm{kg}^{-1}$ & 230 & 61 & 65 & 43 & 46 & 41 \\
$\mathrm{~S}$ & $\mathrm{mg} \mathrm{kg}^{-1}$ & 269,8 & 158,7 & 30,7 & 19,6 & 18,8 & 20,1 \\
$\mathrm{~B}$ & $\mathrm{mg} \mathrm{kg}^{-1}$ & 19,61 & 13,07 & 2,29 & 0,65 & 0,33 & $<0,001$ \\
$\mathrm{Cu}$ & $\mathrm{mg} \mathrm{kg}^{-1}$ & 0,49 & 0,18 & 0,13 & 0,09 & 0,08 & 0,07 \\
$\mathrm{Mn}$ & $\mathrm{mg} \mathrm{kg}^{-1}$ & 0,14 & 0,08 & 0,06 & 0,05 & 0,05 & 0,04 \\
$\mathrm{Zn}$ & $\mathrm{mg} \mathrm{kg}^{-1}$ & 0,93 & 0,40 & 0,35 & 0,21 & 0,13 & 0,01 \\
$\mathrm{Fe}$ & $\mathrm{mg} \mathrm{kg}^{-1}$ & 3,30 & 4,40 & 2,40 & 1,40 & 1,60 & 3,00 \\
Densidade & $\mathrm{kg} \mathrm{m}^{-3}$ & 208 & 279 & 402 & 475 & 586 & 684 \\
$\mathrm{C} / \mathrm{N}$ & - & 80,2 & 47,6 & 32,3 & 36,8 & 25,6 & 17,4 \\
$\mathrm{CE}$ & $\mathrm{mS} \mathrm{m}^{-1}$ & 152,1 & 716,7 & 559,5 & 425,4 & 447,5 & 414,8 \\
\hline
\end{tabular}

T0 - 100\% substrato base; T1 - $80 \%$ substrato base + 20\% composto orgânico; T2 - $60 \%$ substrato base + 40\% composto orgânico; T3 - 40\% substrato base + 60\% composto orgânico; T4 - 20\% substrato base + 80\% composto orgânico; T5 - 100\% composto orgânico. C/ $\mathrm{N}$ - Relação carbono/nitrogênio. CE - Condutividade elétrica

matéria seca da parte aérea) e PSA/PSR (relação entre o peso da matéria seca da parte aérea e o peso da matéria seca da raiz), além das características morfológicas $\mathrm{H}, \mathrm{D}$, número de folhas (NF), PSA (peso da matéria seca da parte aérea), PSR (peso da matéria seca da raiz) e PST (peso de matéria seca total).

Para a medição do peso da matéria seca foram mensuradas 25 plantas por tratamento, sendo cinco plantas de cada repetição com o menor desvio padrão em relação à média do diâmetro da repetição. Para a medição do peso a planta foi dividida em parte aérea e raiz. As raízes foram lavadas em água para a separação do substrato; posteriormente, o material foi colocado em sacos de papel pardo e postos para secar em estufa de circulação forçada de ar a temperatura de $65^{\circ} \mathrm{C}+/-3$, até atingir peso constante.

Os dados dos experimentos passaram por análise das pressuposições básicas estatísticas, averiguando a normalidade e a homogeneidade de variância (a 5\% de probabilidade), sendo a transformação de dados realizada, quando necessário, pelo método de potência ótima de Box-Cox; em seguida, os tratamentos foram submetidos à análise de variância pelo teste F $(\alpha=5 \%)$.

Com os dados de $\mathrm{H}$ e $\mathrm{D}$ foram geradas curvas de crescimento, através de análise de regressão e índice de determinação ajustado, para avaliação das taxas de crescimento das mudas. Os índices e as características morfológicas das mudas avaliadas aos 120 dias após a semeadura (DAS) foram analisados com o teste de
Scott-Knott a 0,05 de significância. As análises dos dados foram realizadas pelo software $R$.

\section{Resultados e Discussão}

Ao final do experimento observou-se, aos 120 dias após a semeadura, que os tratamentos que apresentaram mudas com maiores médias de altura foram o T3 e T4, com 60 e $80 \%$ de composto na composição, respectivamente (Tabela 3). Os tratamentos T0, T1 e T5 apresentaram menores médias. Gomes et al. (2002) afirmaram, estudando características morfológicas na avaliação da qualidade de mudas de Eucalyptus grandis, que a altura deve estar com uma média de aproximadamente $30 \mathrm{~cm}$ para ser considerada apta ao plantio; percebe-se, então, que a maioria dos tratamentos testados se encontra próximo a esta média porém Wendling \& Dutra (2010) defendem que o valor mínimo para o plantio de mudas de eucalipto no campo é de 15 $\mathrm{cm}$. Com base nesta ótica, todos os tratamentos apresentaram médias de altura superiores a este mínimo. Na literatura se encontram estudos de produção de mudas de eucalipto, de 90 a 120 dias de ciclo de produção, apresentando médias de altura superiores aos $15 \mathrm{~cm}$ e, eventualmente, acima dos $20 \mathrm{~cm}$ (Bazzo, 2009; Pelissari et al., 2009; Kratz et al., 2013).

Observa-se, em relação à variável $\mathrm{D}$, grande semelhança com as respostas obtidas para $\mathrm{H}$. Os maiores valores obtidos

Tabela 3. Médias das características morfológicas e índices medidos aos 120 dias após a semeadura das mudas de Eucalyptus "urograndis" em viveiro

\begin{tabular}{cccccccc}
\hline Tratamentos & T0 & T1 & T2 & T3 & T4 & T5 & CV (\%) \\
H $(\mathrm{cm})$ & $(18,730) \mathrm{c}$ & $(20,106) \mathrm{c}$ & $(29,342) \mathrm{b}$ & $(37,310) \mathrm{a}$ & $(33,658) \mathrm{a}$ & $(22,170) \mathrm{c}$ & 11,23 \\
D $(\mathrm{mm})$ & $(2,502) \mathrm{d}$ & $(2,520) \mathrm{d}$ & $(2,836) \mathrm{c}$ & $(3,412) \mathrm{a}$ & $(3,080) \mathrm{b}$ & $(2,646) \mathrm{d}$ & 5,58 \\
H/D & $(7,498) \mathrm{b}$ & $(7,974) \mathrm{b}$ & $(10,326) \mathrm{a}$ & $(10,904) \mathrm{a}$ & $(10,950) \mathrm{a}$ & $(8,372) \mathrm{b}$ & 7,99 \\
NF & $(45,730) \mathrm{b}$ & $(52,442) \mathrm{a}$ & $(43,524) \mathrm{b}$ & $(40,574) \mathrm{b}$ & $(33,890) \mathrm{c}$ & $(26,594) \mathrm{d}$ & 13,14 \\
PST $(\mathrm{g})$ & $(1,430) \mathrm{c}$ & $(1,502) \mathrm{c}$ & $(1,766) \mathrm{b}$ & $(2,314) \mathrm{a}$ & $(2,158) \mathrm{a}$ & $(1,380) \mathrm{c}$ & 10,83 \\
PSA (g) & $(0,960) \mathrm{c}$ & $(0,938) \mathrm{c}$ & $(1,140) \mathrm{b}$ & $(1,528) \mathrm{a}$ & $(1,448) \mathrm{a}$ & $(0,882) \mathrm{c}$ & 11,38 \\
PSR $(\mathrm{g})$ & $(0,470) \mathrm{c}$ & $(0,562) \mathrm{c}$ & $(0,630) \mathrm{b}$ & $(0,786) \mathrm{a}$ & $(0,710) \mathrm{a}$ & $(0,498) \mathrm{c}$ & 12,88 \\
H/PSA & $(19,972) \mathrm{b}$ & $(21,402) \mathrm{b}$ & $(25,900) \mathrm{a}$ & $(24,480) \mathrm{a}$ & $(23,334) \mathrm{a}$ & $(25,206) \mathrm{a}$ & 10,63 \\
PSA/PSR & 2,050 & 1,682 & 1,812 & 1,948 & 2,062 & 1,794 & 11,26 \\
IQD & $(0,150) \mathrm{b}$ & $(0,156) \mathrm{b}$ & $(0,146) \mathrm{b}$ & $(0,178) \mathrm{a}$ & $(0,166) \mathrm{a}$ & $(0,136) \mathrm{b}$ & 9,97 \\
\hline
\end{tabular}

*Variâncias iguais pelo teste $\mathrm{F}(\alpha=5 \%)$. Médias seguidas pela mesma letra minúscula na linha não diferem pelo Teste de Scott-Knott ( $\alpha=5 \%)$. H - Altura, D - diâmetro do colo, H/D - Razão entre altura e diâmetro, NF - número de folhas, PST - peso de matéria seca total, PSA - peso da matéria seca da parte aérea, PSR - peso da matéria seca da raiz, H/PSA - relação entre a altura e 0 peso da matéria seca da parte aérea, PSA/PSR - relação entre o peso da matéria seca da parte aérea e 0 peso da matéria seca da raiz, IQD - índice de qualidade de Dickson. T0 - 100\% substrato base; T1 - 80\% substrato base + 20\% composto orgânico; T2 - 60\% substrato base + 40\% composto orgânico; T3 - 40\% substrato base + 60\% composto orgânico; T4 - 20\% substrato base $+80 \%$ composto orgânico; T5 - 100\% composto orgânico. CV\% - Coeficiente de variação 
para o $\mathrm{D}$, assim como para $\mathrm{H}$, foram os tratamentos $\mathrm{T} 3 \mathrm{e} \mathrm{T} 4$, porém apresentando diferença estatística entre as médias ( $\mathrm{p}<0,05)$, diferentemente do ocorrido para a H. De acordo com Carneiro (1995) o diâmetro é amplamente utilizado para indicar a capacidade de adaptação da muda no campo, refletindo sobre seu potencial de sobrevivência. Gomes et al. (1996) e Wendling \& Dutra (2010) propuseram 2 mm como limite mínimo do D para considerar uma muda de eucalipto bem formada. Desta forma, todos os tratamentos estudados se encontram acima do limite mínimo aos 120 DAS. Em alguns estudos de produção de mudas de eucalipto, de 90 a 120 dias de ciclo de produção, foram constatadas médias de D superiores aos 1,30 mm e, eventualmente, acima dos 1,60 mm (Bazzo, 2009; Pelissari, et al., 2009; Kratz et al., 2013).

Para a relação $H / D$ verificaram-se os maiores valores para as mudas dos tratamentos T4, T3 e T2. De acordo com Carneiro (1995) esta relação é importante para exprimir o equilíbrio do crescimento da muda no viveiro, porque ela conjuga dois fatores em um único índice, evitando equívocos em avaliar a qualidade da muda com essas variáveis isoladamente. De acordo com o mesmo autor, a faixa deste índice considerada ótima, se encontra entre 5,4 e 8,1. Assim, todos os tratamentos se encontram acima do valor máximo; entretanto, é preciso salientar que Carneiro (1995) calculou esses índices em estudos realizados com Pinus taeda nos quais foram fixados um diâmetro mínimo de $3,7 \mathrm{~mm}$ e uma altura de muda de 20 a $30 \mathrm{~cm}$. Recalculando esta relação com o diâmetro mínimo utilizado no presente trabalho, de $2 \mathrm{~mm}$ e as mesmas alturas, obteve-se uma faixa ótima de H/D variando de 10 a 15; desta maneira, os tratamentos T2, T3 e T4 se encontram dentro dessa nova faixa ótima. Kratz et al. (2013) e Trigueiro \& Guerrini (2003) obtiveram, estudando a produção de mudas de eucalipto, médias gerais dentro da faixa ótima recalculada, 11,88 e 12,19 respectivamente porém Bazzo (2009) encontrou valores de H/D dentro da faixa apresentada por Carneiro (1995) diferindo dos resultados aqui apresentados.

A variável NF não apresentou uma variação coerente com os diferentes tratamentos propostos nem se alterou como qualquer outra variável avaliada, o que corrobora com os resultados de Binotto (2010) o qual, estudando variáveis de crescimento e desenvolvimento de mudas e o IQD para Pinus e eucalipto, concluiu que a pior variável para se aferir a qualidade das mudas em viveiro foi o NF. É possível que este resultado seja fruto da dificuldade da contagem das folhas em estágios mais avançados da muda, devido ao grande número de folhas e pela eventual perda das mesmas ao longo do tempo (Gonçalves \& Benedetti, 2000). Deve-se, no entanto, frisar que as folhas possuem papel crucial no desenvolvimento vegetal, na produção de fotoassimilados e determinante no uso da água, destacando também o potencial de adaptação das características morfológicas, anatômicas e fisiológicas aos estímulos ambientais influenciando diretamente sua produtividade (Pereira et al., 2013).

Os resultados obtidos no presente experimento para as variáveis PSA, PSR e PST, foram semelhantes sendo que as melhores respostas foram encontradas nos tratamentos T3, T4 e T2, em que os dois primeiros foram superiores ao tratamento $\mathrm{T} 2$, demonstrando a resposta das plantas ao aumento da quantidade de composto no substrato. A média do PST está próxima dos limites citados por Gonçalves \& Benedetti (2000), os quais citam que a matéria seca para Eucalyptus grandis aos 97 dias após a semeadura se encontra entre 1,2 e 1,6 g. Tendo em conta que o presente estudo foi conduzido com uma espécie híbrida de Eucalyptus grandis X Eucalyptus urophylla e que o ciclo de produção de mudas teve duração de 120 dias, é aceitável que esses valores se encontrem pouco acima da faixa citada anteriormente.

Os mesmos autores complementam que o total acumulado do PSA e PSR se encontra entre $70-75 \%$ e 25 - 30\% do PST respectivamente. No presente estudo os valores de PSA e PSR estão fora da faixa citada e o PSA se mantém entre 65 - 70\% e o PSR entre 30 - 35\%. Resultado semelhante a este experimento, para o PST, foi encontrado no estudo de Trigueiro \& Guerrini (2003), porém com valores de PSR e PSA distante dos indicados por Gonçalves \& Benedetti (2000).

$\mathrm{O}$ índice PSA/PSR teve as variâncias consideradas iguais ( $\mathrm{p}>0,05)$, não apresentando diferença entre as médias dos tratamentos. Realmente, percebe-se que as médias de todos os tratamentos para esta variável estão próximas de 2 (Tabela 3). Caldeira et al. (2000) citam que o valor de PSA/PSR para mudas de eucalipto deve ser 2 , ou 0,5 calculando-se a razão de forma inversa. Deste modo, percebe-se um equilíbrio entre as massas na formação das mudas desse experimento.

O IQD é considerado boa medida morfológica integrada levando em consideração diversas características importantes, como PST, PSA, PSR, H e D, conferindo a robustez e o equilíbrio da distribuição das massas (Binotto, 2010). De forma geral, quanto maior seu valor melhor a qualidade da muda. Para este índice as mudas que apresentaram os melhores resultados estavam presentes nos tratamentos T3 e T4. O IQD médio geral para o experimento foi de 0,155 . Resultado bastante próximo ao do presente estudo foi apresentado por Kratz et al. (2013) e Steffen et al. (2011), os quais estudando diferentes espécies de Eucalyptus e Corymbia encontraram médias de IQD entre 0,16 e 0,17 , respectivamente.

Gomes \& Paiva (2004) defendem que o valor mínimo do IQD deve ser de 0,20; Desta maneira, todos os exemplos aqui citados foram dos padrões para esses autores. Deve-se atentar para o fato desse índice ter sido calculado para as espécies Pseudotsuga menziessi e Picea abies, e, desta forma, este valor pode não representar o gênero Eucalyptus com precisão. Além do mais, os valores de IQD podem sofrer variações entre as próprias espécies de eucalipto tal como com o ciclo de produção de mudas.

Visando explorar e melhor entender as respostas dos tratamentos para as características D e H em função da idade, foram ajustadas equações de retas, as quais descreveram melhor o fenômeno de crescimento nas condições testadas.

Os tratamentos T3, T4 e T2 (60, 80 e 40\% de composto) possuíram as maiores médias e coeficientes angulares das retas, representando maiores taxas de incremento da $\mathrm{H}$ em função do tempo, mesmo apresentando erros padrões altos e coeficientes de determinação ajustado baixo (Tabela 4).

Os resultados obtidos para as retas de crescimento do D tiveram semelhança com as apresentadas para $\mathrm{H}$, tendo como diferença valores mais baixos dos erros padrões e 
Tabela 4. Incremento em altura da parte aérea (H) e diâmetro do colo (D) das mudas de eucalipto no viveiro

\begin{tabular}{|c|c|c|c|c|c|c|}
\hline \multirow{2}{*}{ Tratamento } & Equação & Erro padrão & $\mathrm{R}^{2}$ ajust (\%) & Equação & Erro padrão & $\mathrm{R}^{2}$ ajust (\%) \\
\hline & & Altura & & \multicolumn{3}{|c|}{ Diâmetro } \\
\hline T0 & $-3,755^{\star}+0,18087^{\star} x($ dias $)$ & 1,332 & 95,61 & $0,02170 \star x$ (dias) & 0,1993 & 88,30 \\
\hline T1 & $-3,319^{\star}+0,18712^{\star} \times($ dias $)$ & 1,302 & 96,06 & $0,02200 * x$ (dias) & 0,1390 & 94,13 \\
\hline T2 & $-7,703^{\star}+0,28329^{\star} x($ dias $)$ & 3,332 & 89,48 & $-0,534333^{\star}+0,027567^{\star} \times$ (dias) & 0,1589 & 95,07 \\
\hline T3 & $-10,797^{\star}+0,37257^{\star} x($ dias $)$ & 4.385 & 89,47 & $-0,496667^{\star}+0,032133^{*} \times$ (dias) & 0,1647 & 96,06 \\
\hline T4 & $-10,271^{\star}+0,34328^{\star} x$ (dias) & 3,003 & 93,91 & $-0,51800^{\star}+0,029800 * x($ dias $)$ & 0,1270 & 97,25 \\
\hline T5 & $-5,957^{\star}+0,22353^{\star} x($ dias $)$ & 2,516 & 90,28 & $-0,713667^{*}+0,027300 * x$ (dias) & 0,1862 & 93,22 \\
\hline
\end{tabular}

$\mathrm{R}^{2}$ ajust. - Coeficiente de determinação ajustado. T0 - 100\% substrato base; T1 - 80\% substrato base $+20 \%$ composto orgânico; T2 - 60\% substrato base $+40 \%$ composto orgânico; T3 - 40\% substrato base + 60\% composto orgânico; T4 - 20\% substrato base + 80\% composto orgânico; T5 - 100\% composto orgânico; * significativo a 1\% de probabilidade pelo teste F

coeficientes de determinação ajustados mais altos; desta forma, as melhores respostas se encontram nos tratamentos T3, T4 e T2 (Tabela 4).

A resposta para este comportamento está intimamente ligada à característica física dos substratos, considerada muito importante para a formação de mudas, aqui representada pela densidade. De acordo com Lopes et al. (2008) a faixa considerada ótima para esta variável se encontra entre 450 e $550 \mathrm{~kg} \mathrm{~m}^{-3}$, variando próximo aos tratamentos $\mathrm{T} 2, \mathrm{~T} 3 \mathrm{e} \mathrm{T} 4$ tendo, como destaque, o tratamento T3, o qual se encontra dentro da faixa citada acima. Esses resultados estão de acordo com os obtidos por Sabonaro et al. (2009) os quais perceberam, estudando a produção de mudas de Tabebuia heptaphylla em diferentes substratos, que as maiores alturas da parte aérea e do diâmetro do colo foram obtidas nos substratos com densidade entre 470 e $500 \mathrm{~kg} \mathrm{~m}^{-3}$.

De forma semelhante, Bazzo (2009) obteve, estudando a utilização de composto orgânico de lodo de esgoto na formação de mudas de eucalipto, as melhores respostas de crescimento em relação ao $\mathrm{D}$ no tratamento controle, o qual possuiu as maiores alturas; já na análise dos tratamentos que continham a mistura de composto orgânico com casca de arroz carbonizado, as melhores respostas do diâmetro foram as proporções 20:80 e 60:40, que também apresentaram as maiores alturas.

\section{Conclusão}

A qualidade das mudas de Eucalyptus "urograndis" produzidas em substratos contendo composto orgânico nas proporções 40:60 e 20:80 (substrato base:composto orgânico), proveniente de resíduos de fábrica de papel e celulose, mostrouse superior à qualidade das mudas produzidas com o substrato base de controle, de acordo com as variáveis avaliadas.

\section{Literatura Citada}

ABRAF - Associação Brasileira de Produtores de Florestas Plantadas. Anuário estatístico da ABRAF 2013 ano base 2012. Brasília: ABRAF, 2013.148p.

Assenheimer, A. Benefícios do uso de biossólidos como substratos na produção de mudas de espécies florestais. Ambiência, v.5, p.321-330, 2009.

Barretto, V. C. M. Resíduos de indústria de celulose e papel na fertilidade do solo e no desenvolvimento de eucalipto. Jaboticabal: UNESP, 2008. 64p. Tese Doutorado

Bazzo, J. F. Utilização de composto orgânico de lodo de esgoto como substrato para produção de mudas de eucalipto. Botucatu: UNESP, 2009. 62p. Dissertação Mestrado
Binotto, A. F. Correlations between growth variables and the Dickson quality index in forest seedlings. Cerne, v.16, p.457-464, 2010. http://dx.doi.org/10.1590/S0104-77602010000400005

BRACELPA - Associação Brasileira de Celulose e Papel. Dados do setor, outubro - 2013, 2013. 5p.

Caldeira, M. V. W.; Schumacher, M. V.; Barrichello, L. R.; Voget, H. L. M.; Oliveira, L. S. Crescimento de mudas de Eucalyptus saligna Smith em função de diferentes doses de vermicomposto. Floresta, v.28, p.19-30, 2000.

Carneiro, J. G. A. Produção e controle de qualidade de mudas florestais. Curitiba: FUPEF/UFPR, 1995. 451p.

Daudt, R. H. S.; Gruszynski, C.; Kämpf, A. N. Uso de resíduos de couro wet-blue como componente de substrato para plantas. Ciência Rural, v.37, p.91-96, 2007. http://dx.doi.org/10.1590/ S0103-84782007000100015

Freitas, T. A. S.; Barroso, D. G.; Souza, L. S.; Carneiro, J. G. A.; Paulino, G. M. Produção de mudas de eucalipto com substratos para sistema de blocos. Revista Árvore, v.34, p.761-770, 2010. http:// dx.doi.org/10.1590/S0100-67622010000500001

Galbiatti, J. A.; Lui, J. J.; Sabonaro, D. Z.; Bueno, L. F.; Silva, V. L. Formação de mudas de eucalipto com utilização de lixo orgânico e níveis de irrigação calculados por dois métodos. Engenharia Agrícola, v.27, p.445-455, 2007. http://dx.doi.org/10.1590/S010069162007000300013

Gomes, J. M.; Couto, L.; Leite, H. G.; Xavier, A.; Garcia, S. L. R. Parâmetros morfológicos na avaliação da qualidade de mudas de Eucalyptus grandis. Revista Árvore, v.26, p.655-664, 2002. http:// dx.doi.org/10.1590/S0100-67622002000600002

Gomes, J. M.; Paiva, H. N. Viveiros florestais: Propagação sexuada. 3.ed. Viçosa: UFV, 2004. 116p.

Gomes, J. M.; Paiva, H. N.; Couto, L. Produção de mudas de eucalipto. Informe Agropecuário, v.18, p.15-22, 1996.

Gonçalves, J. L. M.; Benedetti, V. Nutrição e fertilização florestal. Piracicaba: IPEF, 2000. 427p.

Gonçalves, J. L. M.; Stape, J. L. Conservação e cultivo de solos para plantações florestais. Piracicaba: IPEF, 2002. 498p.

Kratz, D.; Wendling, I.; Nogueira, A. C.; Souza, P. V. D. Substratos renováveis na produção de mudas de Eucalyptus benthamii. Ciência Florestal, v.23, p.607-621, 2013. http://dx.doi. org/10.5902/1980509812345

Lima, J. D.; Moraes, W. S.; Mendonça, J. C.; Nomura, E. S. Resíduos da agroindústria de chá preto como substrato para produção de mudas de hortaliças. Ciência Rural, v.37, p.1609-1613, 2007. http://dx.doi.org/10.1590/S0103-84782007000600016

Lopes, J. L.W.; Guerrini, I. A.; Saad, J. C. C.; Silva, M. R. Atributos químicos e físicos de dois substratos para produção de mudas de eucalipto. Revista Cerne, v.14, p.358-367, 2008. 
Maeda, S.; Dedecek, R. A.; Agostine, R. B.; Andrade, G. C.; Silva, H. D. Caracterização de substratos para a produção de mudas de espécies florestais elaborados a partir de resíduos orgânicos. Pesquisa Florestal Brasileira, v.54, p.97-104, 2007.

Mendonça, A. V. R.; Carneiro, J. G. A.; Barroso, D. G.; Santiago, A. R.; Freitas, T. A. S.; Souza, J. S. Desempenho de quatro espécies de Eucalyptus spp em plantios puros e consorciados com sabiá (Mimosa caesalpiniaefolia Benth) em cava de extração de argila. Revista Árvore, v.32, p.395-405, 2008. http://dx.doi.org/10.1590/ S0100-67622008000300002

Paiva, A. V.; Poggiani, F.; Gonçalves, J. L. M.; Ferraz, A. V. Crescimento de mudas de espécies arbóreas nativas, adubadas com diferentes doses de lodo de esgoto seco e com fertilização mineral. Scientia Forestalis, v.37, p.499-511, 2009.

Pelissari, R. A. Z.; Sampaio, S. C.; Gomes, S. D.; Crepalli, M. S. Lodo têxtil e água residuária da suinocultura na produção de mudas de Eucalyptus grandis (W, Hill ex Maiden). Engenharia Agrícola, v.29, p.288-300, 2009. http://dx.doi.org/10.1590/S010069162009000200012

Pereira, T. A. R.; Silva, L. C.; Azevedo, A. A.; Francino, D. M. T.; Coser, T. S.; Pereira, J. D. Leaf morpho-anatomical variations in Billbergia elegans and Neoregelia mucugensis (Bromeliaceae) exposed to low and high solar radiation. Botany, v.91, p.327-334, 2013. http:// dx.doi.org/10.1139/cjb-2012-0276
Ribeiro, H. M.; Romero, A. M.; Pereira, H.; Borges, P.; Cabral, F; Vasconcelos, E. Evaluation of a compost obtained from forestry wastes and solid phase of pig slurry as a substrate for seedlings production. Bioresource Technology, v.98, p.3294-3297, 2007. http://dx.doi.org/10.1016/j.biortech.2006.07.002

Sabonaro, D. Z.; Galbiatti, J. A.; Paula, R. C.; Gonzales, J. L. S. Producción de plantas de Tabebuia heptaphylla en diferentes sustratos y niveles de irrigación, en condiciones de invernadero. Bosque, v.30, p.27-35, 2009.

Santos, F. E. V.; Araújo, J. M.; Andrade, W. C.; Costa, C. C.; Silva, A. G. Formação de mudas de Eucalyptus urophylla S.T. Blake com utilização de resíduo sólido orgânico urbano. Enciclopédia Biosfera, v.9, p.1203-1214, 2013.

Steffen, G. P. K.; Antoniolli, Z. I.; Steffen, R. B.; Schiedeck, G. Utilização de vermicomposto como substrato na produção de Eucalyptus grandis e Corymbia citriodora. Pesquisa Florestal Brasileira, v.31, p.75-82, 2011. http://dx.doi.org/10.4336/2011. pfb.31.66.75

Trigueiro R. M.; Guerrini, I. A. Uso de biossólido como substrato para produção de mudas de eucalipto. Scientia Forestalis, n.64, p.150-162, 2003.

Wendling, I.; Dutra, L. F. Produção de mudas de eucalipto por sementes. In: Wendling, I.; Dutra, L. F. Produção de mudas de eucalipto. Colombo: Embrapa Florestas, 2010. p.13-47. 